\title{
Article \\ Patisiran in hATTR Amyloidosis: Six-Month Latency Period
before Efficacy
}

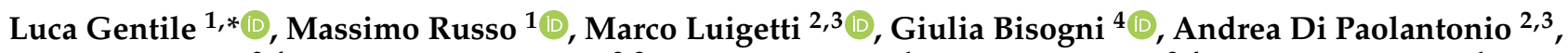 \\ Angela Romano ${ }^{2,4}$, Valeria Guglielmino ${ }^{2,3}$, Ilenia Arimatea ${ }^{1}$, Mario Sabatelli ${ }^{2,4}$, Antonio Toscano ${ }^{1}$, \\ Giuseppe Vita ${ }^{1}$ and Anna Mazzeo ${ }^{1}$
}

1 Unit of Neurology and Neuromuscular Diseases, Department of Clinical and Experimental Medicine, University of Messina, 98125 Messina, Italy; russom@unime.it (M.R.); ilenia.arimatea@hotmail.it (I.A.); atoscano@unime.it (A.T.); giuseppe.vitag@unime.it (G.V.); annamazzeo@yahoo.it (A.M.)

2 UOC Neurologia, Fondazione Policlinico Universitario A. Gemelli IRCCS, 00168 Roma, Italy; mluigetti@gmail.com (M.L.); andrea.dp1988@gmail.com (A.D.P.); angela.romano12@gmail.com (A.R.); guglielmino.valeria@gmail.com (V.G.); mario.sabatelli@unicatt.it (M.S.)

3 Dipartimento di Neuroscienze, Università, Cattolica del Sacro, Cuore, 00168 Roma, Italy

4 Centro Clinico NEMO, Fondazione Policlinico Universitario A. Gemelli IRCCS, 00168 Roma, Italy; giulia.bisogni@centrocliniconemo.it

* Correspondence: lucagentile84@yahoo.it; Tel.: +39-090-2213-504

\section{check for}

updates

Citation: Gentile, L.; Russo, M.; Luigetti, M.; Bisogni, G.; Di

Paolantonio, A.; Romano, A.;

Guglielmino, V.; Arimatea, I.;

Sabatelli, M.; Toscano, A.; et al.

Patisiran in hATTR Amyloidosis: Six-Month Latency Period before Efficacy. Brain Sci. 2021, 11, 515. https: / / doi.org/10.3390/

brainsci11040515

Academic Editor: Thomas H Hutson

Received: 24 March 2021

Accepted: 16 April 2021

Published: 19 April 2021

Publisher's Note: MDPI stays neutral with regard to jurisdictional claims in published maps and institutional affiliations.

Copyright: (c) 2021 by the authors. Licensee MDPI, Basel, Switzerland. This article is an open access article distributed under the terms and conditions of the Creative Commons Attribution (CC BY) license (https:// creativecommons.org/licenses/by/ $4.0 /)$.

\begin{abstract}
Hereditary amyloidosis associated with mutations in the transthyretin gene (hATTR) is a progressive devastating disease, with a fatal outcome occurring within 10years after onset. In recent years, TTR gene silencing therapy appeared as a promising therapeutic strategy, showing evidence that disease progression can be slowed and perhaps reversed. We report here 18 subjects affected by hATTR amyloidosis treated with patisiran, a small interfering RNA acting as TTR silencer, and evaluated with a PND score, the NIS and NIS-LL scale, and a Norfolk QOL-DN questionnaire at baseline and then every 6 months. A global clinical stabilizationwas observed for the majority of the patients, with mild-moderate improvements in some cases, even in advanced disease stage (PND score $>2$ ). Analysis of NIS, NIS-LL and Norfolk QOL-DN results, and PND score variation suggest the possible presence of a 6-month latency period prior to benefit of treatment.
\end{abstract}

Keywords: hATTR; amyloidosis; TTR silencers; gene therapy

\section{Introduction}

Hereditary amyloidosis associated with mutations in the transthyretin (TTR) gene (hATTR) is the most common form of genetic amyloidosis. It is a progressive devastating disease transmitted as an autosomal dominant trait, with a fatal outcome occurring within 10years after onset [1,2]. More than 130 TTR gene mutations have been identified thus far, with Val30Met as the most common mutation reported worldwide [3-5]. The liver is the primary source of circulating tetrameric TTR, but it is also synthesized by the retinal pigment epithelium and choroid plexus. TTR functions primarily as a transport protein for vitamin A and for approximately 15\% of circulating plasma thyroxine. In patients with hATTR amyloidosis, the tetramer dissociates in dimers and, then, in monomers, which deposit in target tissues forming abnormal amyloid fibril aggregates [6]. However, in hATTR amyloidosis, both mutant and wild-type TTR deposit as amyloid in peripheral nerves and in many other organs, including heart, kidney, and gastrointestinal tract [6-8]. While the exact clinical phenotype depends on the underlying mutation, cardiomyopathy, peripheral polyneuropathy, and autonomic neuropathy with orthostatic hypotension and gastrointestinal dysautonomia are common [9], and the previously used definition of Familial Amiloidotic Cardiomyopathy (FAC) or Familial AmiloidoticPolineuropathy (FAP) have become outdated $[10,11]$. The necessity to monitor disease progression has recently led to an increase of the research on new biomarkers [12-18], with the particular aim to 
record the shift from asymptomatic to symptomatic stage, whichcould allow physicians to start specific treatment for hATTR amyloidosis.

Liver transplantation (LT) and combined heart-liver transplantation represented the first specific therapy for hATTR amyloidosis, suppressing the main source of mutant TTR. However, its effectiveness has been demonstrated mainly in Val30Met patients and can be influenced by pre-treatment patient's features (disease duration, nutritional status, age, severity of neuropathy, and cardiac involvement) [19]. In 2011, tafamidis, an orally administered TTR protein stabilizer, was approved by European Medicine Agency on the basis of its results in study Fx-005 (NCT00409175), a pivotal placebo-controlled study, which enrolled patients with Stage 1 disease and the Val30Met mutation in the TTR gene. Although published data showed that tafamidis is able to delay the course of neuropathy with good preservation of nutritional status, a neurologic progression has been observed in $40-65 \%$ of patients after 12 months of tafamidis treatment [20-22]. In more recent years, TTR gene silencing therapy with an antisense oligonucleotide (ASO) (inotersen) or a small interfering RNA (siRNA) (patisiran) appeared as a more promising therapeutic strategy for hATTR amyloidosis. TTR silencers provided a real therapeutic revolution, showing evidence that disease progression can be slowed and perhaps reversed [3,4,9,23-25].

In particular, in the phase 3, randomized, double-blind, placebo-controlled (2:1), 18-month Apollo study, patisiran has been demonstrated effective in substantially reduce TTR concentration [23], with significant and sustained improvement in polyneuropathy scores [26], autonomic neuropathy [27], quality-of-life profile [28], and some cardiac parameters [29]. For some parameters, such as the modified Neuropathy Impairment Score +7 $(\mathrm{mNIS}+7)$ and the Norfolk Quality of Life-Diabetic Neuropathy questionnaire total score (Norfolk QOL-DN), a statistically significant improvement was achieved at 18 months compared with placebo, with effects seen as early as 9 months [3]. The interim 12-month analysis of the ongoing lobal open-label extension (OLE) study, which involved patients from the Apollo study and from the 24-month phase 2 single-arm OLE, patisiran appeared to maintain long-term efficacy with an acceptable safety profile [30,31]. Since July 2018, this drug has been available in Italy for patients as compassionate use.

\section{Patients and Methods}

\subsection{Patients and Outcome Measures}

We report here 18 subjects affected by hATTR amyloidosis and treated with patisiran. They were evaluated at the "Regional Centre of Reference for Diagnosis and Treatment of Amyloidosis" of the Unit of Neurology and Neuromuscular Diseases-Department of Clinical and Experimental Medicine-University of Messina (Italy) or at the "Fondazione PoliclinicoUniversitario, A. Gemelli IRCCS," Unit of Neurology, Largo A Gemelli 8, 00168 Rome, Italy. All 18 subjects underwent neurologic evaluation, each obtaining a Polyneuropathy Disability (PND) score (with higher scores indicating more impaired walking ability), aNeuropathy Impairment Score (NIS) (range, 0 to 192, with higher scores indicating more impairment), and a quality of life assessment with the Norfolk Quality of Life-Diabetic Neuropathy (Norfolk QOL-DN) questionnaire (range, -4 to 136, with higher scores indicating worse quality of life) at baseline and after 6 months of treatment (M6). Fifteen patients were evaluated after 12 months (M12) and 9 patients after 18 months (M18). For these three items, we also recorded retrospective data in all patients at 6, 12, and 18 months before baseline (M-6, M-12, and M-18).

Improvement in the NIS score was defined as a reduction of at least 3 points; the increaseof at least 3 points in this score was considered a sign of clinical worsening. Patients with an NIS score variation between \pm 2.75 points were considered stable. Similarly, improvement in the Norfolk QOL-DN score was defined as a reduction of at least 4 points; an increaseof at least 4 points in this score was considered a sign of worsening quality of life. Patients with a Norfolk QOL-DN score variation between \pm 3 points were considered stable.

This study was approved and performed under the ethical guidelines issued by our institutions for clinical studies and was in compliance with the Helsinki Declaration. The 
study was approved by the ethics committee of our hospitals, and informed written consent was obtained from all the patients(Ethical Committee Code:nr.3/2016, of the 22 march 2016. Name: Ethical Committee of the University Hospital of Messina (address: AOU “G.Martino," via Consolare Valeria n.1, 98125-Messina (ME), Italy)).

\subsection{Statistical Analysis}

For the purpose of statistical analysis, the patients were divided into sixgroups based on the length of the available observation period before and after treatment, on the presence of a co-treatment with tafamids and on the PND stage at baseline:

- $\quad$ Group 1 (all patients) from M-6 to M6.

- $\quad$ Group 2 (14 patients) from M-12 to M12.

- $\quad$ Group 3 (11 patients) from M-18 to M18.

- $\quad$ Group 4 (4 patients with tafamidis as concomitant medication) from M-12 to M12.

- $\quad$ Group 5 (8 patients in PND stage 1-2) from M-12 to M12.

- $\quad$ Group 6 (6 patients in PND stage 3A and 3B) from M-12 to M12.

The statistical analysis was performed by calculating the mean value and the one sample Wilcoxon test between the deltas of the pre-treatment period and those of the post-treatment period in the sixabove-mentioned groups. Statistical significance was set at $p<0.05$ (lowered after Bonferroni's adjustment to <0.008).

\section{Results}

\subsection{Demographics}

The 18 patients harbored six different TTR mutations: Phe64Leu (n.7), Glu89Gln (n.5), Val30Met (n.2), Thr49Ala (n.2), Val122Ile (n.1), and Ala109Ser (n.1) (Table 1). Age at onset varied from 45 to 75 years (mean: 59.6), with hATTR amyloidosis diagnosis set 2.6 years after first symptoms (mean age at diagnosis: 62.2 years). Mean age at first patisiran infusion was 63.8 years. Four patients were also in treatment with tafamidismeglumine; because of poor response, patisiranwas initiatedwithout withdrawing tafamidis, as per the indications of thecompassionate useprogram.

Table 1. Demographics, genotypes and concomitant treatments.

\begin{tabular}{|c|c|c|c|c|c|}
\hline Patient & Mutation & Age at Onset & $\begin{array}{c}\text { Age at } \\
\text { Diagnosis }\end{array}$ & $\begin{array}{c}\text { Age at } \\
\text { Baseline }\end{array}$ & $\begin{array}{c}\text { Concomitant } \\
\text { Treatments }\end{array}$ \\
\hline pt. CP & Phe64Leu & 68 & 72 & 78 & $\backslash$ \\
\hline pt. GM (d) ${ }^{1}$ & Phe64Leu & 63 & 68 & 68 & 1 \\
\hline pt. SC & Ala109Ser & 75 & 71 & 75 & 1 \\
\hline pt. SA & Val30Met & 59 & 62 & 68 & 1 \\
\hline pt. TG & Phe64Leu & 48 & 54 & 55 & Tafamidis \\
\hline pt. CF & Glu89Gln & 48 & 54 & 55 & Tafamidis \\
\hline pt. MS & Phe64Leu & 61 & 66 & 67 & Tafamidis \\
\hline pt. AU & Phe64Leu & 75 & 77 & 77 & 1 \\
\hline pt. RC (d) ${ }^{1}$ & Val122Ile & 65 & 69 & 70 & 1 \\
\hline pt. TMG & Glu89Gln & 56 & 57 & 58 & 1 \\
\hline pt. BG & Phe64Leu & 71 & 73 & 74 & Tafamidis \\
\hline pt. CS & Phe64Leu & 66 & 68 & 69 & 1 \\
\hline pt. VG & Glu89Gln & 48 & 50 & 50 & 1 \\
\hline pt. VA & Val30Met & 71 & 75 & 75 & 1 \\
\hline pt. MA & Glu89Gln & 45 & 46 & 47 & 1 \\
\hline pt. PA & Thr49Ala & 55 & 56 & 57 & 1 \\
\hline pt. MMA & Thr49Ala & 46 & 47 & 50 & 1 \\
\hline pt. RB (d) ${ }^{1}$ & Glu89Gln & 54 & 55 & 57 & 1 \\
\hline
\end{tabular}

(d) ${ }^{1}$ : deceased. 


\subsection{Neurologic Evaluation}

At baseline, PND score ranged from 1 to $3 \mathrm{~b}$ (Table A1). The same result was obtained at last follow up, except for threepatients who improved (two from 3 a to 2 , and one from $3 \mathrm{~b}$ to 2 ) and twopatients who worsened (one from class 2 to $3 a$ and one from class 2 to $3 b)$. The data of the patient who presented the best response to patisiran treatment were previously published [32].

Mean NIS showed a progressive increase in the 18 months before starting patisiran, being 70.2 at baseline. This parameter continued to worsen after the first 6 months of treatment (18/18 patients mean NIS at M6: 72) (Figure 1). An improvement started after 12 months (15/18 patients mean NIS at M12: 70.9), persisting after 18 months (11/18 patients mean NIS at M18: 68). Globally, 7/18 (38.8\%) patients improved at NIS, 6/18 (33.3\%) remained stable, and 5/18 (27.7\%) worsened (Table A2).

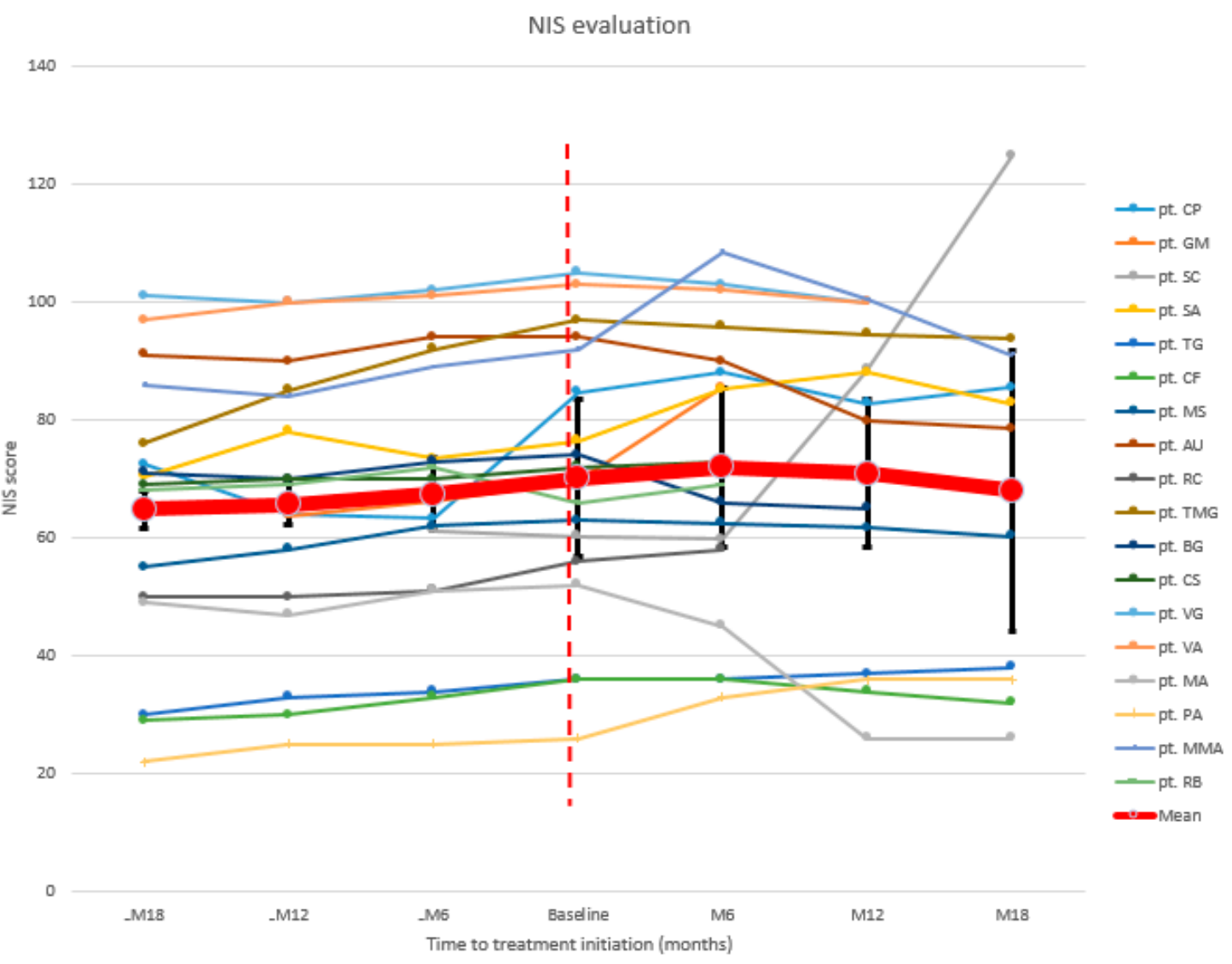

Figure 1. NIS: neuropathy impairment score.

Similar results were found at NIS lower limb (NIS-LL), a subscale of NIS obtained considering only lower limb motor strength, reflexes, and sensitivity data (range 0-88) (Figure 2). After a continuing increase in the months before, mean NIS-LL was 39.8 at baseline, and worsened after 6 months (18/18 patients mean NIS-LL at M6: 41.4). At the 12-month evaluation, NIS-LL was returned to baseline levels (15/18 patients mean NIS-LL at M12: 39.9) with a considerable improvement registered after 18 months (11/18 patients mean NIS-LL at M18: 37.1). Globally, 6/18 (33.3\%) patients improved at NIS-LL, 6/18 $(33.3 \%)$ remained stable, and 6/18 (33.3\%) worsened (Table A3). 


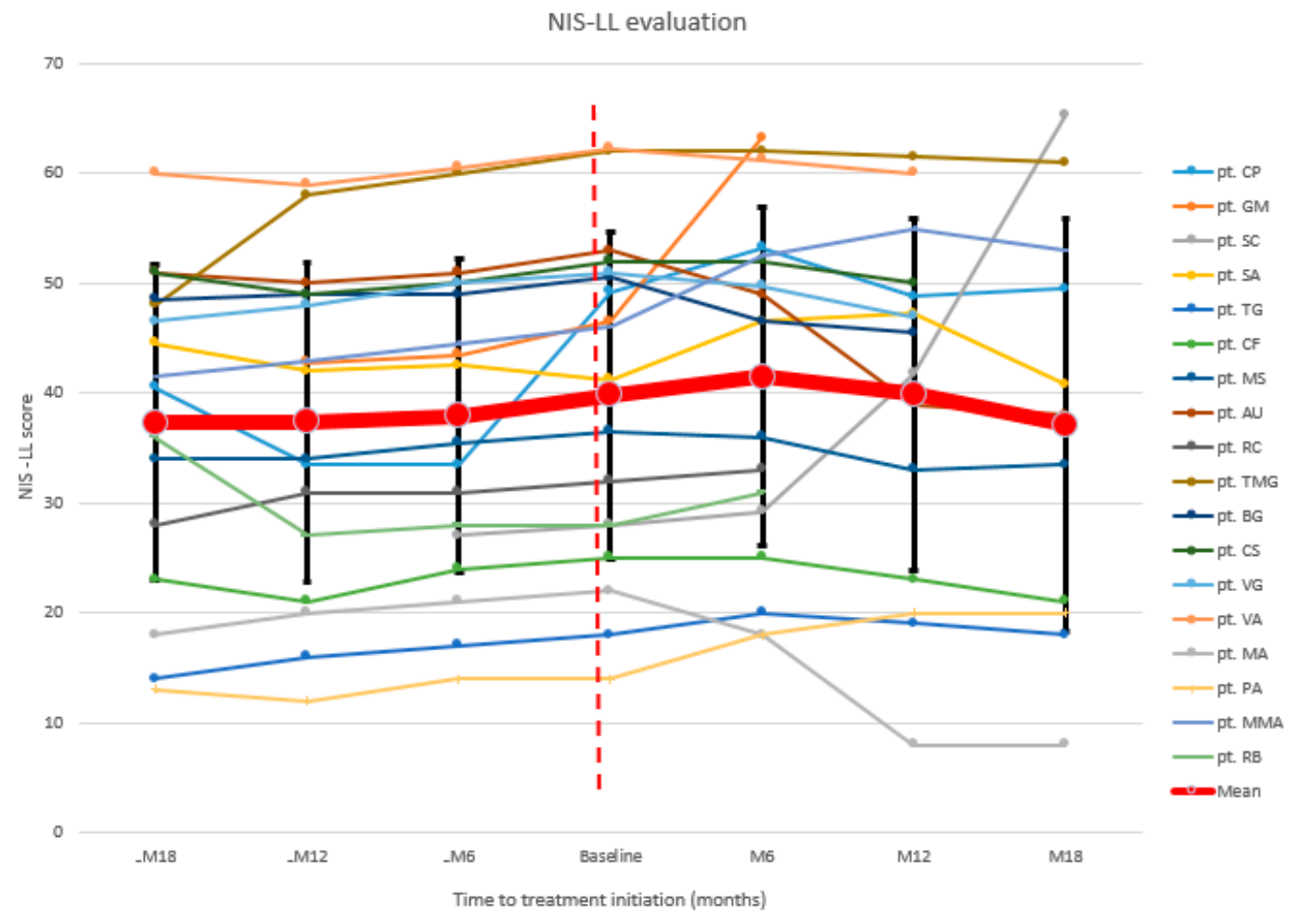

Figure 2. NIS-LL: neuropathy impairment score lower limbs.

The results of the statistical analyses for NIS and NIS-LL are shown in Table 2. To facilitate the comparison of the deltas between groups (with different features and different duration of observation), the values are expressed as mean monthly change. Comparing the pre-treatment deltas vs post treatment deltas, a significant difference was observed in all subgroups except for group 4. Table 2 includes mean monthly change in NIS-LL obtained in the placebo group and in the tafamidis group in the tafamidis trial [33].

Table 2. NIS and NS-LL statistical analysis.

\begin{tabular}{cccc}
\hline Group of Patients & \multicolumn{2}{c}{ Mean Monthly Change } \\
& Pre-Treatment & Post-Tratment & $p$ \\
G.1-NIS & 0.46 & 0.31 & $\mathbf{0 . 0 0 4}$ \\
G.2-NIS & 0.43 & -0.15 & $\mathbf{0 . 0 0 0}$ \\
G.3-NIS & 0.42 & -0.19 & $\mathbf{0 . 0 0 2}$ \\
G.4-NIS & 0.38 & -0.23 & 0.125 \\
G.5-NIS & 0.88 & -0.24 & 0.015 \\
G.6-NIS & 0.97 & -0.67 & 0.031 \\
G. 1-NIS-LL & 0.32 & 0.27 & $\mathbf{0 . 0 0 1}$ \\
G. 2-NIS-LL & 0.27 & -0.11 & $\mathbf{0 . 0 0 0}$ \\
G. 3-NIS-LL & 0.22 & -0.13 & $\mathbf{0 . 0 0 2}$ \\
G. 4-NIS-LL & 0.21 & -0.20 & 0.125 \\
G. 5-NIS-LL & 0.64 & -0.19 & 0.015 \\
G. 6-NIS-LL & 0.49 & -0.46 & 0.031 \\
Placebo group NIS-LL [33] & & \\
Tafamidis Group NIS-LL [33] & 0.16 & 0.32 & \\
\hline G. = Group; G.1 $=$ all patients from M-6 to M6; G.2 = 14 patients from M-12 to M12; G.3 = 10 patients from M-18 to \\
M18; G.4 = 4 patients with tafamidis as concomitant medication from M-12 to M12; G.5 = 8 patients in PND stage \\
1-2 from M-12 to M12; G.6 = 6 patients in PND stage 3A and 3B from M-12 to M12. In bold, significantvalues.
\end{tabular}

\subsection{Quality of Life Assessment}

To assessquality of life, we recorded data from 17/18 patients. Mean Norfolk QOL-DN continued to worsen in the 18 months before patisiran and also after 6 months of treatment 
(17/17 patients' mean Norfolk QOL-DN at baseline: 64.2; at M6: 65.7) (Figure 3). A decrease was recorded after 12 months (15/17 patients mean value at M12: 62.7), as well as after 18 months (11/17 patients mean value at M18: 57.4$)$. Globally, 10/17 (58.8\%) patients improved at Norfolk QOL-DN, 4/17 (23.5\%) remained stable, and 3/17 (17.6\%) worsened (Table A4).

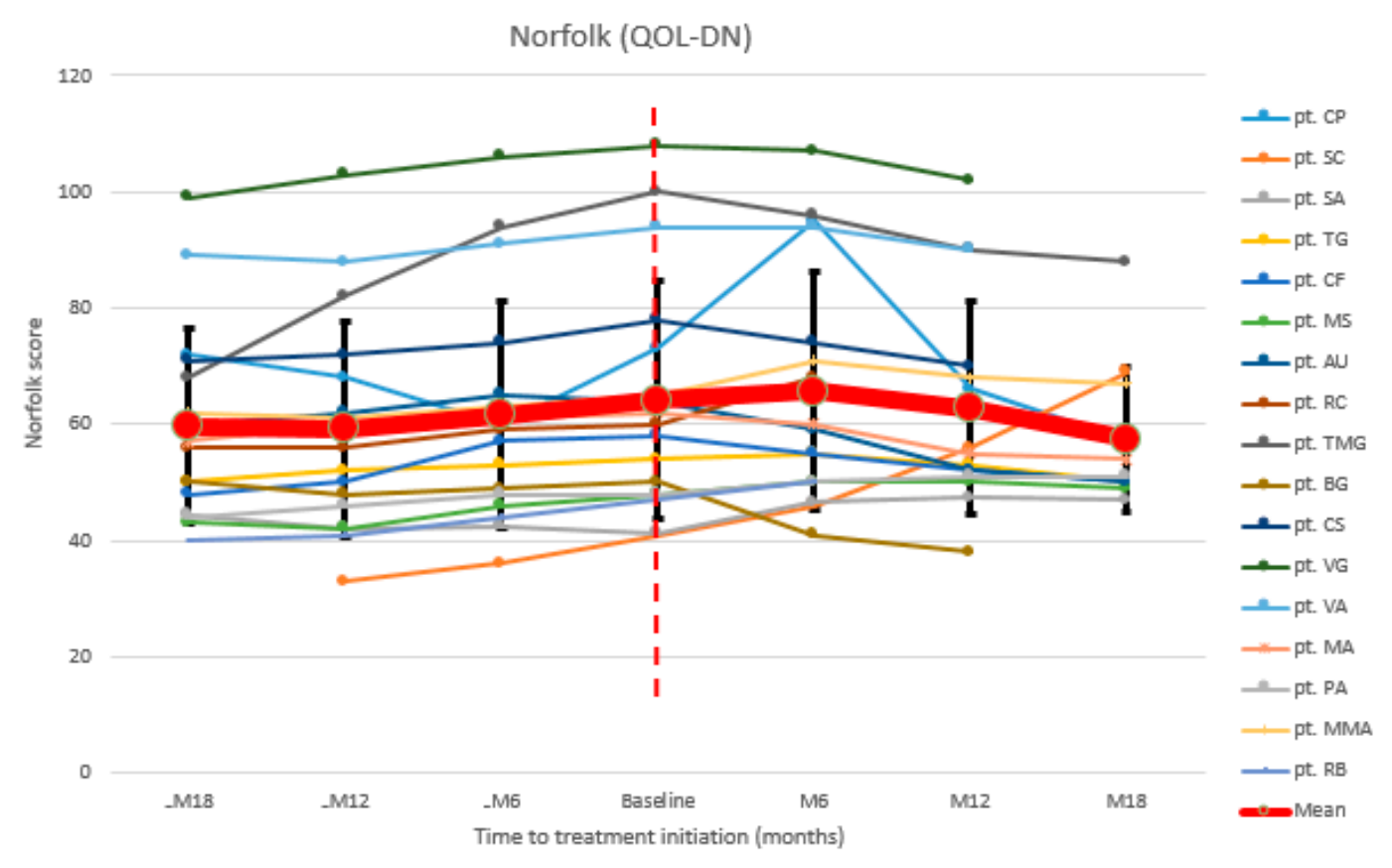

Figure 3. Norfolk QOL-DN: Norfolk quality of life diabetic neuropathy.

The results of the statistical analysis for this scale are shown in Table 3. As for NIS and NIS-LL, no significant difference was found in group4. Instead, a significant difference was observed when comparing the deltas in all the remaining five groups.

Table 3. Norfolk QoL statistical analysis.

\begin{tabular}{cccc}
\hline Group of Patients & \multicolumn{2}{c}{ Mean Monthly Change } & \\
& Pre-Treatment & Post-Tratment & \\
\hline G. 1-Norfolk Qol & 0.42 & 0.26 & $\mathbf{0 . 0 0 2}$ \\
G. 2-Norfolk Qol & 0.37 & -0.27 & $\mathbf{0 . 0 0 1}$ \\
G. 3-Norfolk Qol & 0.36 & -0.28 & $\mathbf{0 . 0 0 1}$ \\
G. 4-Norfolk Qol & 0.38 & -0.35 & 0.125 \\
G. 5-Norfolk Qol & 0.63 & -0.29 & 0.023 \\
G. 6-Norfolk Qol & 1.03 & -1.25 & 0.031 \\
\hline
\end{tabular}

G. = Group; G.1 = all patients from M-6 to M6; G.2 = 14 patients from M-12 to M12; G.3 = 10 patients from M-18 to M18; G.4 = 4 patients with tafamidis as concomitant medication from M-12 to M12; G.5 = 8 patients in PND stage 1-2 from M-12 to M12; G.6 = 6 patients in PND stage 3A and 3B from M-12 to M12. In bold, significant values.

\subsection{Adverse Events}

We reported two side effects secondary to the use of premedication drugs: hyperglycemia in one patient (maximum fasting value of $361 \mathrm{mg} / \mathrm{dL}$ ) and hypertension in two patients (maximum systolic value of $180 \mathrm{mmHg}$ and diastolic of $125 \mathrm{mmHg}$ ). These conditions improved after appropriate drug therapy.

Three patients temporarily discontinued the therapy because of hospitalizations, for severe anemia, fever (two distinct episodes), and diarrhea, respectively. All patients started the therapy again after their discharge. 
We also reported three deaths. Two patients presented a sudden death, probably of cardiac origin, after 9 and 16 months of therapy, respectively. Another patient died of acomplication aftersevere dehydration.

\section{Discussion}

Real-world, post-marketing, observational studies can provide useful information beyond the confines of traditional clinical trials. This is particularly true when multiple drugs are available and clinicians need to better understand individual indication criteria.

Eighteen hATTR amyloidosis patients, with different genotype and phenotype, were treated with patisiran for a mean period of 14 months. A stabilization of disease progression, with a significant improvement in some cases, expressed by the NIS, NIS-LL, Norfolk QOL$\mathrm{DN}$, and PND score variation, was observed.

PND score remained stable in $13 / 18$ patients and improved in 3/18. Interestingly, none of the $7 / 18$ patients that were in stage $3 a$ or $3 b$ showed a worsened PND score: four of them remained in the same PND class and three moved to a lower class, expressing a better walking capacity (Table A1). However, in this subgroup of patients, we did not finda significant difference between NIS, NIS-LL, and Norfolk QOL-DN changes before norafter the beginning of treatment (although an analysis showed a $p<0.05$, whichlost significance after Bonferroni's adjustment). These results, suggesting the possible presence of a promising efficacy of patisiran even in late stages of disease, need to be further confirmed in larger group of patients. Instead, considering the whole cohort, the analysis of NIS and NIS-LL results showed a global clinical improvement, with a considerable reduction of these scores seen at 18-month follow-up visits (Tables 2, A2 and A3). One limitation of the present study is that only 11/18 patients completed the M18 evaluation, but an improving tendency was already clear at M12 (milestone reached by 15/18 patients) (Table 2). On the other hand, at M6, both mean NIS and NIS-LL monthly changes $(+0.31$ and +0.27 , respectively) suggested a continuation of the progressive clinical worsening registered from M-18 to baseline, although a significant difference was found between the deltas pre- and post-treatment, even in this group of patients (Table 2). In the Apollo study, clinical improvement (represented by change from baseline of mNIS+7) was reported after 9 months of treatment. Our data partially confirm what was shown in the Apollo study, since at 12 months, there wasa reduction in the NIS and NIS-LL values. However, since no reduction in these scores was observed at M6, thoughthere was a statistically significant slowdown in worsening, these results suggest the possible presence of a 6-month latency period prior to benefit of treatment. The samecould be seenfor the patients' quality of life. Norfolk QOL-DN tended to increase from M-18 to baseline (Table 3, Table A4). The deterioration began to slow significantly in the baseline-M6 period, but we only observed a reduction in the values of this scale in the period M6-M18 (Table 3). Overall, it is not surprising that the worsening of patients' quality of life goes together with their clinical deterioration in the same period of time. As the same, with the improvement of neurologic condition at M12 and M18, a significant decrease of Norfolk QOL-DN was recorded at the same milestones (Table 3). Finally, no significant differences between pre- and posttreatment results were found in the subgroup of patients also treated with tafamidis. This could be explained by the small number of patients in double treatment, whichcould have made such an analysis inconsistent. Adverse events reported were correlated mainly to the use of premedical drugs. The three deaths reported were considered highly unlikely to be related to patisiran infusion.

\section{Conclusions}

Treatment with patisiran is highly recommended in patients with hATTR amyloidosis. The use of outcome measures such as NIS and Norfolk QOL-DN is useful to monitor treatment response, but it should be taken into consideration that a 6-month latency period could be present before clinical benefits become evident. However, further studies involving a greater number of patients should be necessary to confirm these results. 
Author Contributions: Conceptualization, L.G. and M.R.; investigation, L.G., M.R., A.M., M.L. A.D.P., G.B., A.R. and V.G.; data curation, L.G., A.D.P., I.A. and M.L.; writing-original draft preparation, L.G.; writing-review and editing, A.M., A.T., G.V. and M.S. All authors have read and agreed to the published version of the manuscript.

Funding: This research received no external funding.

Institutional Review Board Statement: The study was conducted according to the guidelines of the Declaration of Helsinki, and approved by the Ethics Committee of University Hospital of Messina (Ethical Committee Code: nr.3/2016, of the 22 march 2016).

Informed Consent Statement: Informed consent was obtained from all subjects involved in the study.

Data Availability Statement: The data presented in this study are available on request from the corresponding author.

Conflicts of Interest: L.G. is a sub-investigator in the clinical trials of Alnylam, Ionis, Takeda. He also reports travel grants from Kedrion and CSL Behring to attend scientific meetings and acknowledges a speaker fee and consulting honoraria from Pfizer. M.R.acknowledges receiving speaker fee and consulting honoraria from Akcea and Alnylam and a travel grant from Pfizer.A.M. is the Principal Investigator in the clinical trials of Alnylam and Ionis anda sub-investigator in the clinical trials of Alnylam, Ionis, and Takeda. She also reports travel grants from Kedrion and CSL Behring to attend scientific meetings and acknowledges a speaker fee and consulting honoraria from Alnylam, Akcea, and Pfizer. G.V. is the Principal Investigator in the clinical trials ofRoche, Sarepta, Santhera, Italfarmaco, Biogen, Avexis, Alnylam, Ionis, and Wave. He is also aconsultantof the Advisory Board for Roche, Avexis, Alnylam, and Akcea and he acknowledges a speaker fee and consulting honoraria from Alnylam, Akcea, and Pfizer. A.T. is the Principal Investigator in the clinical trials of Takeda and Genzyme. He acknowledges a speaker fee and consulting honoraria from Kedrion, CSL Behring, and Genzyme. M.L. received financial grants (honoraria and speaking) from Ackea, Alnylam, and Pfizer, and travel grants from Ackea, Alnylam, Pfizer, Kedrion, Csl Behring, and Grifols. A.R. received travel grants from Pfizer and Csl Behring and financial grants from Akcea. A.D.P. received travel grants from Pfizer. G.B. received financial grants (honoraria and speaking) from Alnylam and travel grants from Pfizer, Alnylam, and Grifols. V.G. has no potential conflicts of interest to disclose.

\section{Appendix A}

Table A1. PND score results.

\begin{tabular}{ccc}
\hline Patient & PND at Baseline & PND at Last Follow-Up \\
\hline pt. CP & 2 & $3 \mathrm{a}$ \\
pt. GM (d) $)^{2}$ & 2 & 2 \\
pt. SC & 2 & $3 \mathrm{~b}$ \\
pt. SA & 2 & 2 \\
pt. TG & 1 & 1 \\
pt. CF & 1 & 1 \\
pt. MS & 2 & 2 \\
pt. AU & $3 \mathrm{~b}$ & 2 \\
pt. RC (d) & $3 \mathrm{~b}$ & $3 \mathrm{~b}$ \\
pt. TMG & $3 \mathrm{a}$ & 2 \\
pt. BG & $3 \mathrm{a}$ & 2 \\
pt. CS & $3 \mathrm{~b}$ & $3 \mathrm{~b}$ \\
pt. VG & $3 \mathrm{a}$ & $3 \mathrm{a}$ \\
pt. VA & 2 & 2 \\
pt. MA & 2 & 2 \\
pt. PA & 1 & 1 \\
pt. MMA & $3 \mathrm{a}$ & $3 \mathrm{a}$ \\
pt. RB (d) ${ }^{1}$ & 2 & 2 \\
\hline
\end{tabular}

(d) ${ }^{1}$ : deceased. 
Table A2. NIS results.

\begin{tabular}{cccccccc}
\hline Patient & -M18 & -M12 & -M6 & Baseline & M6 & M12 & M18 \\
\hline pt. CP & 72.5 & 64 & 63.25 & 84.75 & 88 & 82.75 & 85.5 \\
pt. GM (d) ${ }^{1}$ & & 63.75 & 66.25 & 69.75 & 85.5 & & \\
pt. SC & & & 61.25 & 60.25 & 59.75 & 88.5 & 124.75 \\
pt. SA & 70.5 & 78 & 73.5 & 76.5 & 85.25 & 88 & 82.75 \\
pt. TG & 30 & 33 & 34 & 36 & 36 & 37 & 38 \\
pt. CF & 29 & 30 & 33 & 36 & 36 & 34 & 32 \\
pt. MS & 55 & 58 & 62 & 63 & 62.5 & 61.75 & 60.25 \\
pt. AU & 91 & 90 & 94 & 94 & 90 & 79.75 & 78.5 \\
pt. RC (d) ${ }^{1}$ & 50 & 50 & 51 & 56 & 58 & & \\
pt. TMG & 76 & 85 & 92 & 97 & 95.75 & 94.5 & 93.75 \\
pt. BG & 71 & 70 & 73 & 74 & 66 & 65 & \\
pt. CS & 69 & 70 & 70 & 72 & 73 & 70 & \\
pt. VG & 101 & 100 & 102 & 105 & 103 & 100 & \\
pt. VA & 97 & 100 & 101 & 103 & 102 & 100 & \\
pt. MA & 49 & 47 & 51 & 52 & 45 & 26 & 26 \\
pt. PA & 22 & 25 & 25 & 26 & 33 & 36 & 36 \\
pt. MMA & 86 & 84 & 89 & 92 & 108.5 & 100.5 & 91 \\
pt. RB (d) ${ }^{1}$ & 68 & 69 & 72 & 66 & 69 & & \\
Mean & 64.8 & 65.7 & 67.4 & 70.2 & 72 & 70.9 & 68 \\
\hline (d) ${ }^{1 .}$ deceased & & & & & & &
\end{tabular}

(d) ${ }^{1}$ : deceased.

Table A3. NIS-LL results.

\begin{tabular}{cccccccc}
\hline Patient & -M18 & -M12 & -M6 & Baseline & M6 & M12 & M18 \\
\hline pt. CP & 40.5 & 33.5 & 33.5 & 49.25 & 53.25 & 48.75 & 49.5 \\
pt. GM (d) & & 42.75 & 43.5 & 46.5 & 63.25 & & \\
pt. SC & & & 27 & 28 & 29.25 & 41.7 & 65.25 \\
pt. SA & 44.5 & 42 & 42.5 & 41.25 & 46.5 & 47.25 & 40.75 \\
pt. TG & 14 & 16 & 17 & 18 & 20 & 19 & 18 \\
pt. CF & 23 & 21 & 24 & 25 & 25 & 23 & 21 \\
pt. MS & 34 & 34 & 35.5 & 36.5 & 36 & 33 & 33.5 \\
pt. AU & 51 & 50 & 51 & 53 & 49 & 39 & 38 \\
pt. RC (d) & 28 & 31 & 31 & 32 & 33 & & \\
pt. TMG & 48 & 58 & 60 & 62 & 62 & 61.5 & 61 \\
pt. BG & 48.5 & 49 & 49 & 50.5 & 46.5 & 45.5 & \\
pt. CS & 51 & 49 & 50 & 52 & 52 & 50 & \\
pt. VG & 46.5 & 48 & 50 & 51 & 49.75 & 47 & \\
pt. VA & 60 & 59 & 60.5 & 62.25 & 61.25 & 60 & \\
pt. MA & 18 & 20 & 21 & 22 & 18 & 8 & 8 \\
pt. PA & 13 & 12 & 14 & 14 & 18 & 20 & 20 \\
pt. MMA & 41.5 & 43 & 44.5 & 46 & 52.5 & 55 & 53 \\
pt. RB (d) ${ }^{1}$ & 36 & 27 & 28 & 28 & 31 & & \\
Mean & 37.3 & 37.4 & 37.9 & 39.8 & 41.4 & 39.9 & 37.1 \\
\hline
\end{tabular}

(d) ${ }^{1}$ : deceased. 
Table A4. Norfolk QOL-DN results.

\begin{tabular}{|c|c|c|c|c|c|c|c|}
\hline Patient & -M18 & -M12 & -M6 & Baseline & M6 & M12 & M18 \\
\hline pt. CP & 72 & 68 & 60 & 73 & 95 & 66 & 57 \\
\hline pt. SC & & 33 & 36 & 41 & 46 & 56 & 69 \\
\hline pt. SA & 44.5 & 42 & 42.5 & 41.25 & 46.5 & 47.25 & 47 \\
\hline pt. TG & 50 & 52 & 53 & 54 & 55 & 53 & 50 \\
\hline pt. CF & 48 & 50 & 57 & 58 & 55 & 52 & 50 \\
\hline pt. MS & 43 & 42 & 46 & 48 & 50 & 50 & 49 \\
\hline pt. AU & 60 & 62 & 65 & 64 & 59 & 52 & 50 \\
\hline pt. $\mathrm{RC}(\mathrm{d})^{1}$ & 56 & 56 & 59 & 60 & 68 & & \\
\hline pt. TMG & 68 & 82 & 94 & 100 & 96 & 90 & 88 \\
\hline pt. BG & 50 & 48 & 49 & 50 & 41 & 38 & \\
\hline pt. CS & 71 & 72 & 74 & 78 & 74 & 70 & \\
\hline pt. VG & 99 & 103 & 106 & 108 & 107 & 102 & \\
\hline pt. VA & 89 & 88 & 91 & 94 & 94 & 90 & \\
\hline pt. MA & 57 & 60 & 61 & 62 & 60 & 55 & 54 \\
\hline pt. PA & 44 & 46 & 48 & 48 & 50 & 51 & 51 \\
\hline pt. MMA & 62 & 61 & 63 & 65 & 71 & 68 & 67 \\
\hline pt. $\mathrm{RB}(\mathrm{d})^{1}$ & 40 & 41 & 44 & 47 & 50 & & \\
\hline Mean & 59.6 & 59.2 & 61.7 & 64.2 & 65.7 & 62.7 & 57.4 \\
\hline
\end{tabular}

(d) ${ }^{1}$ : deceased.

\section{References}

1. Ando, Y.; Coelho, T.; Berk, J.L.; Cruz, M.W.; Ericzon, B.-G.; Ikeda, S.-I.; Lewis, W.D.; Obici, L.; Planté-Bordeneuve, V.; Rapezzi, C.; et al. Guideline of transthyretin-related hereditary amyloidosis for clinicians. Orphanet J. Rare Dis. 2013, 8, 31. [CrossRef]

2. Mazzeo, A.; Russo, M.; Di Bella, G.; Minutoli, F.; Stancanelli, C.; Gentile, L.; Baldari, S.; Carerj, S.; Toscano, A.; Vita, G. Transthyretin-Related Familial Amyloid Polyneuropathy (TTR-FAP): A Single-Center Experience in Sicily, an Italian Endemic Area. J. Neuromuscul. Dis. 2015, 2, S39-S48. [CrossRef] [PubMed]

3. Gertz, M.A.; Mauermann, M.L.; Grogan, M.; Coelho, T. Advances in the treatment of hereditary transthyretin amyloidosis: A review. Brain Behav. 2019, 9, e01371. [CrossRef] [PubMed]

4. Kristen, A.V.; Ajroud-Driss, S.; Conceição, I.; Gorevic, P.; Kyriakides, T.; Obici, L. Patisiran, an RNAi therapeutic for the treatment of hereditary transthyretin-mediated amyloidosis. Neurodegener. Dis. Manag. 2019, 9, 5-23. [CrossRef]

5. Russo, M.; Obici, L.; Bartolomei, I.; Cappelli, F.; Luigetti, M.; Fenu, S.; Cavallaro, T.; Chiappini, M.G.; Gemelli, C.; Pradotto, L.G.; et al. ATTRv amyloidosis Italian Registry: Clinical and epidemiological data. Amyloid 2020, 27, 259-265. [CrossRef]

6. Planté-Bordeneuve, V.; Said, G. Familial amyloid polyneuropathy. Lancet Neurol. 2011, 10, 1086-1097. [CrossRef]

7. Russo, M.; Mazzeo, A.; Stancanelli, C.; Di Leo, R.; Gentile, L.; Di Bella, G.; Minutoli, F.; Baldari, S.; Vita, G. Transthyretin-related familial amyloidotic polyneuropathy: Description of a cohort of patients with Leu64 mutation and late onset. J. Peripher. Nerv. Syst. 2012, 17, 385-390. [CrossRef] [PubMed]

8. Luigetti, M.; Romozzi, M.; Bisogni, G.; Cardellini, D.; Cavallaro, T.; Di Paolantonio, A.; Fabrizi, G.M.; Fenu, S.; Gentile, L.; Grandis, M.; et al. hATTR Pathology: Nerve Biopsy Results from Italian Referral Centers. Brain Sci. 2020, 10, 780. [CrossRef]

9. Müller, M.L.; Butler, J.; Heidecker, B. Emerging therapies in transthyretin amyloidosis-A new wave of hope after years of stagnancy? Eur. J. Heart Fail. 2020, 22, 39-53. [CrossRef]

10. Gentile, L.; Di Bella, G.; Minutoli, F.; Cucinotta, F.; Obici, L.; Mussinelli, R.; Arimatea, I.; Russo, M.; Toscano, A.; Vita, G.; et al. Description of a large cohort of Caucasian patients with V122I ATTRv amyloidosis: Neurological and cardiological features. J. Peripher. Nerv. Syst. 2020, 25, 273-278. [CrossRef]

11. Stancanelli, C.; Gentile, L.; Di Bella, G.; Minutoli, F.; Russo, M.; Vita, G.; Mazzeo, A. Phenotypic variability of TTR Val122Ile mutation: A Caucasian patient with axonal neuropathy and normal heart. Neurol. Sci. 2016, 38, 525-526. [CrossRef] [PubMed]

12. Louwsma, J.; Brunger, A.F.; Bijzet, J.; Kroesen, B.J.; Roeloffzen, W.W.H.; Bischof, A.; Kuhle, J.; Drost, G.; Lange, F.; Kuks, J.B.M.; et al. Neurofilament light chain, a biomarker for polyneuropathy in systemic amyloidosis. Amyloid 2021, 28, 50-55. [CrossRef]

13. Ticau, S.; Sridharan, G.V.; Tsour, S.; Cantley, W.L.; Chan, A.; Gilbert, J.A.; Erbe, D.; Aldinc, E.; Reilly, M.M.; Adams, D.; et al. Neurofilament Light Chain as a Biomarker of Hereditary Transthyretin-Mediated Amyloidosis. Neurology 2021, 96, e412-e422. [CrossRef] [PubMed]

14. Vita, G.L.; Aguennouz, M.; Polito, F.; Oteri, R.; Russo, M.; Gentile, L.; Barbagallo, C.; Ragusa, M.; Rodolico, C.; Di Giorgio, R.M.; et al. Circulating microRNAs Profile in Patients with Transthyretin Variant Amyloidosis. Front. Mol. Neurosci. 2020, $13,102$. [CrossRef] [PubMed]

15. Salvalaggio, A.; Coraci, D.; Cacciavillani, M.; Obici, L.; Mazzeo, A.; Luigetti, M.; Pastorelli, F.; Grandis, M.; Cavallaro, T.; Bisogni, G.; et al. Nerve ultrasound in hereditary transthyretin amyloidosis: Red flags and possible progression biomarkers. J. Neurol. 2021, 268, 189-198. [CrossRef] 
16. Vita, G.L.; Stancanelli, C.; Gentile, L.; Barcellona, C.; Russo, M.; Di Bella, G.; Vita, G.; Mazzeo, A. 6MWT performance correlates with peripheral neuropathy but not with cardiac involvement in patients with hereditary transthyretin amyloidosis (hATTR). Neuromuscul. Disord. 2019, 29, 213-220. [CrossRef]

17. Okada, M.; Misumi, Y.; Masuda, T.; Takashio, S.; Tasaki, M.; Matsushita, H.; Ueda, A.; Inoue, Y.; Nomura, T.; Nakajima, M.; et al. Plasma growth differentiation factor 15: A novel tool to detect early changes of hereditary transthyretin amyloidosis. ESC Heart Fail. 2020, 8, 1178-1185. [CrossRef]

18. Minutoli, F.; Di Bella, G.; Mazzeo, A.; Laudicella, R.; Gentile, L.; Russo, M.; Vita, G.; Baldari, S. Serial scanning with 99mTc-3, 3-diphosphono-1, 2-propanodicarboxylic acid (99mTc-DPD) for early detection of cardiac amyloid deposition and prediction of clinical worsening in subjects carrying a transthyretin gene mutation. J. Nucl. Cardiol. 2019, 1-9. [CrossRef]

19. Ericzon, B.-G.; Wilczek, H.E.; Larsson, M.; Wijayatunga, P.; Stangou, A.J.; Pena, J.R.; Furtado, E.; Barroso, E.; Daniel, J.M.; Samuel, D.; et al. Liver Transplantation for Hereditary Transthyretin Amyloidosis. Transplantation 2015, 99, 1847-1854. [CrossRef]

20. Vita, G.; Vita, G.L.; Stancanelli, C.; Gentile, L.; Russo, M.; Mazzeo, A. Genetic neuromuscular disorders: Living the era of a therapeutic revolution. Part 1: Peripheral neuropathies. Neurol. Sci. 2019, 40, 661-669. [CrossRef] [PubMed]

21. Planté-Bordeneuve, V.; Lin, H.; Gollob, J.; Agarwal, S.; Betts, M.; Fahrbach, K.; Chitnis, M.; Polydefkis, M. An indirect treatment comparison of the efficacy of patisiran and tafamidis for the treatment of hereditary transthyretin-mediated amyloidosis with polyneuropathy. Expert Opin. Pharmacother. 2018, 20, 473-481. [CrossRef]

22. Cortese, A.; Vita, G.; Luigetti, M.; Russo, M.; Bisogni, G.; Sabatelli, M.; Manganelli, F.; Santoro, L.; Cavallaro, T.; Fabrizi, G.M.; et al. Monitoring effectiveness and safety of Tafamidis in transthyretin amyloidosis in Italy: A longitudinal multicenter study in a non-endemic area. J. Neurol. 2016, 263, 916-924. [CrossRef] [PubMed]

23. Adams, D.; Gonzalez-Duarte, A.; O’Riordan, W.D.; Yang, C.-C.; Ueda, M.; Kristen, A.V.; Tournev, I.; Schmidt, H.H.; Coelho, T.; Berk, J.L.; et al. Patisiran, an RNAi Therapeutic, for Hereditary Transthyretin Amyloidosis. N. Engl. J. Med. 2018, $379,11-21$. [CrossRef] [PubMed]

24. Benson, M.D.; Waddington-Cruz, M.; Berk, J.L.; Polydefkis, M.; Dyck, P.J.; Wang, A.K.; Planté-Bordeneuve, V.; Barroso, F.A.; Merlini, G.; Obici, L.; et al. Inotersen Treatment for Patients with Hereditary Transthyretin Amyloidosis. N. Engl. J. Med. 2018, 379, 22-31. [CrossRef]

25. Russo, M.; Gentile, L.; Toscano, A.; Aguennouz, M.; Vita, G.; Mazzeo, A. Advances in Treatment of ATTRv Amyloidosis: State of the Art and Future Prospects. Brain Sci. 2020, 10, 952. [CrossRef] [PubMed]

26. Milani, P.; Mussinelli, R.; Perlini, S.; Palladini, G.; Obici, L. An evaluation of patisiran: A viable treatment option for transthyretinrelated hereditary amyloidosis. Expert Opin. Pharmacother. 2019, 20, 2223-2228. [CrossRef]

27. González-Duarte, A.; Berk, J.L.; Quan, D.; Mauermann, M.L.; Schmidt, H.H.; Polydefkis, M.; Waddington-Cruz, M.; Ueda, M.; Conceição, I.M.; Kristen, A.V.; et al. Analysis of autonomic outcomes in APOLLO, a phase III trial of the RNAi therapeutic patisiran in patients with hereditary transthyretin-mediated amyloidosis. J. Neurol. 2019, 267, 703-712. [CrossRef]

28. Obici, L.; Berk, J.L.; González-Duarte, A.; Coelho, T.; Gillmore, J.; Schmidt, H.H.-J.; Schilling, M.; Yamashita, T.; Labeyrie, C.; Iii, T.H.B.; et al. Quality of life outcomes in APOLLO, the phase 3 trial of the RNAi therapeutic patisiran in patients with hereditary transthyretin-mediated amyloidosis. Amyloid 2020, 27, 153-162. [CrossRef]

29. Solomon, S.D.; Adams, D.; Kristen, A.; Grogan, M.; González-Duarte, A.; Maurer, M.S.; Merlini, G.; Damy, T.; Slama, M.S.; Brannagan, T.H.; et al. Effects of Patisiran, an RNA Interference Therapeutic, on Cardiac Parameters in Patients With Hereditary Transthyretin-Mediated Amyloidosis. Circulation 2019, 139, 431-443. [CrossRef]

30. Adams, D.; Polydefkis, M.; González-Duarte, A.; Wixner, J.; Kristen, A.V.; Schmidt, H.H.; Berk, J.L.; López, I.A.L.; Dispenzieri, A.; Quan, D.; et al. Long-term safety and efficacy of patisiran for hereditary transthyretin-mediated amyloidosis with polyneuropathy: 12-month results of an open-label extension study. Lancet Neurol. 2021, 20, 49-59. [CrossRef]

31. Luigetti, M.; Servidei, S. Patisiran in hereditary transthyretin-mediated amyloidosis. Lancet Neurol. 2021, 20, 21-23. [CrossRef]

32. Russo, M.; Gentile, L.; Toscano, A.; Vita, G.; Mazzeo, A. From a misdiagnosis of anorexia nervosa to a dramatic patisiran-induced improvement in a patient with ATTRE89Q amyloidosis. Amyloid 2020, 27, 279-280. [CrossRef] [PubMed]

33. Coelho, T.; Maia, L.F.; Da Silva, A.M.; Cruz, M.W.; Planté-Bordeneuve, V.; Lozeron, P.; Suhr, O.B.; Campistol, J.M.; Conceição, I.M.; Schmidt, H.H.-J.; et al. Tafamidis for transthyretin familial amyloid polyneuropathy: A randomized, controlled trial. Neurology 2012, 79, 785-792. [CrossRef] 